\title{
THE KINETICS OF CARRIER-MEDIATED TRANSPORT: STATIONARY-STATE APPROXIMATIONS
}

\author{
JOHN A. JACQUEZ \\ Department of Physiology, Medical School, and Department of Biostatistics, \\ School of Public Health, University of Michigan, Ann Arbor, Mich. (U.S.A.) \\ (Received July $5^{\text {th }, ~ 1963) ~}$
}

\begin{abstract}
SUMMARY
The fluxes predicted by various models of carrier-mediated active transport of amino acids have been worked out with use of the assumption of stationarity. The models considered include the various types of models in which there is a I: I carrier-amino acid binding ratio and the limiting cases of this model. The limiting cases are: (I) equilibrium-reaction model: transfers of carrier and carrier-substrate complex across the cell membrane are rate limiting in comparison to the reaction between carrier and amino acid; (2) adsorption transport model: the reaction between carrier and amino acid is rate limiting. The stationary-state fluxes for an equilibrium carrier model in which carrier-amino acid stoichiometry was $I: 2$ were also derived.

The results of experiments on competition between amino acids could not be predicted by models of a $\mathrm{I}$ : I carrier-amino acid stoichiometry unless the assumption was also made that exchange reactions between free and carrier-bound amino acids could occur at the surfaces of the cell membrane. These exchange reactions proceed without prior dissociation of carrier-substrate complex. A model of $I: 2$ carrier-amino acid stoichiometry predicts the results of competition experiments without the necessity of assuming the existance of exchange reactions.
\end{abstract}

\section{INTRODUCTION}

As a preliminary to a computational study of the numerical predictions of various models of active transport of amino acids, I have carried out a systematic examination of the concentration dependence of the fluxes obtained in various carrier models with use of the assumption of stationarity. The results are of considerable interest from the practical viewpoint, particularly since they demonstrate that some of the models commonly used do not fit the experimental findings on amino acids and may therefore be excluded from further consideration.

EXPERIMENTAL CHARACTERISTICS OF THE AMINO ACID ACTIVE TRANSPORT SYSTEM IN EHRLICH ASCITES CELLS

Since the desire to formulate a consistent theoretical framework for the experimental findings reported by various workers for amino acid transport in Ehrlich ascites cells 
motivated this work, a brief review of the major experimental findings follows. Because of this we will speak in terms of amino acid transport but the results are of more general significance.

"Active" transport: Amino acids are in general transported against a concentration gradient by Ehrlich ascites cells and in some cases reach intracellular levels 20-fold higher than the extracellular ${ }^{1-3}$. The uptake of amino acids is partly inhibited by many of the metabolic inhibitors and by anaerobiosis ${ }^{2-4}$.

Competitive relationships: One of the most striking experimental findings is that of the stimulation of the initial flux of one amino acid in the presence of low or approximately equimolar concentrations of some other amino acids ${ }^{3,5,6}$. Thus although alanine inhibits the uptake of tryptophan at all concentrations, the uptake of tryptophan is stimulated by low concentrations of methionine but inhibited by high concentrations of methionine ${ }^{5}$.

Exchange diffusion: The initial flux of an amino acid may be markedly increased if the cells are first loaded with another amino acid ${ }^{5,7-9}$. Preloading with another amino acid may increase or decrease the initial flux of the amino acid under study ${ }^{5}$. This phenomenon has been called counter flow by ROSENBERG AND WILBRANDT ${ }^{10}$.

\section{MODELS OF TRANSPORT}

Since the mathematics involved is trivial, the details will not be presented. In each case the model will be presented and the stationary-state fluxes. In order to obtain the fluxes the differential equations and the conservation equations were set up for each model and then solved for the fluxes under various stationary-state assumptions. In what follows we will generally speak of two amino acids and use $u_{1}$ and $u_{2}$ to represent their extracellular concentrations and $v_{1}$ and $v_{2}$ to represent their respective intracellular concentrations. In each case we derive the stationary-state net flux of amino acid " $I$ " for the following conditions: (a) no other amino acid present; (b) amino acid " 2 " present extracellularly but $v_{1}$ and $v_{2}$ both zero; (c) amino acid " 2 " present in intracellular phase and $v_{1}$ and $u_{2}$ both zero.

These fluxes will be denoted, respectively, $F_{1}\left(u_{1}, v_{1}\right), F_{1}\left(u_{1}, 0, u_{2}, 0\right)$ and $F_{1}\left(u_{1}, 0\right.$, $\left.\mathrm{o}, v_{2}\right)$. Case (b) refers to a hypothetical "competition" experiment in which $v_{1}$ and $v_{2}$ are kept at zero levels and (c) refers to a hypothetical exchange-diffusion experiment in which $v_{1}$ and $u_{2}$ are maintained at zero levels. In all of these cases, the flux due to simple permeability is not included.

\section{Carrier transport models; $I: I$ substrate-carrier stoichiometry}

The general model of this type is shown in Fig. I. Lower-case Greek letters are used to indicate rate constants for the association reactions. The model may be viewed as consisting of two surface phases with transition probabilities for transfer of carrier or of carrier-substrate complexes between the two phases represented by the $k$ 's. We shall call the $k$ 's transfer constants. Some of the predictions of this model have already been published ${ }^{11}$. It is assumed that the total amount of carrier per unit of surface area of the cell membrane is constant, however, we do not assume, as did ROSENBERG AND WILBRANDT ${ }^{12}$, that the total concentration of carrier is equal on the two sides of the membrane.

As was pointed out in a previous paper ${ }^{11}$, carrier active-transport models may 
be classified on the basis of the site of the linkage between the transport system and cellular metabolism. The linkage may occur through one of the four processes, (i) transfer of free carrier between the two surfaces, (ii) transfer of carrier-substrate complex between the two surfaces, (iii) association between carrier and substrate

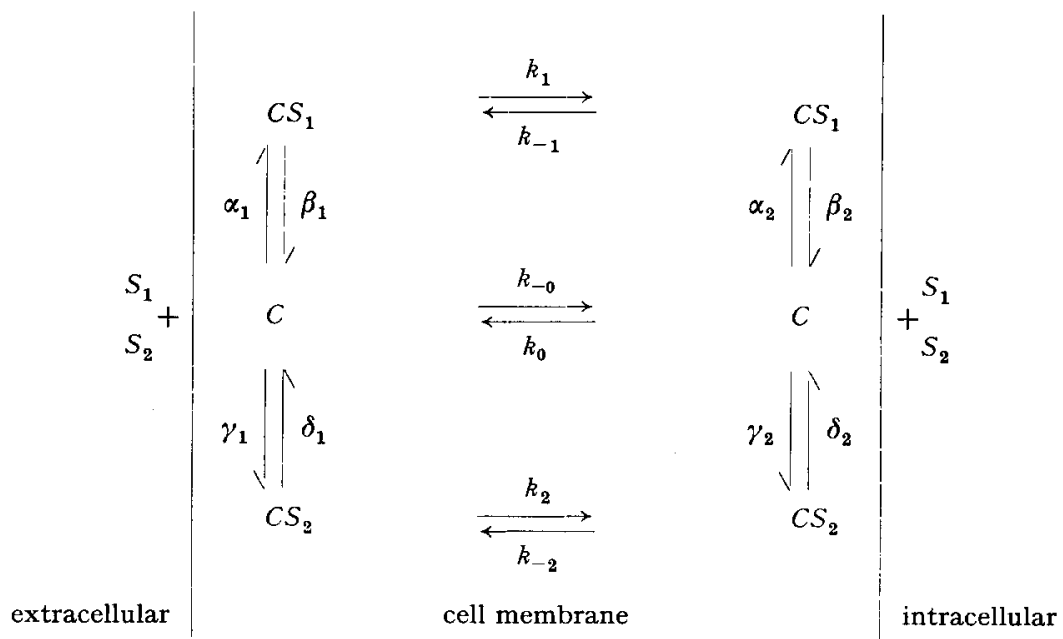

Fig. I. Schematic diagram of general carrier transport model with $\mathrm{I}:$ I substrate-carrier stoichiometry. $S_{1}$ and $S_{2}$ are two different substrates. One-sided arrows are used for chemical reactions, complete arrows represent spatial transfers.

at the outer surface, or (iv) association between carrier and substrate at the inner surface. In order to keep the models as simple as possible, we assume symmetry in rate and transfer constants between the two sides of the cell membrane except for the portion of the transport system which is linked to cellular metabolism. Thus the "active" transport is introduced on a basis ad hoc as an asymmetry in a transfer process or a reaction rate between the two sides of the membrane. Thus the various types of carrier active transport may be characterized as follows (Fig. I).

(I) Active transfer of free carrier:

$$
\begin{aligned}
& \alpha_{1}=\alpha_{2}, \beta_{1}=\beta_{2}, k_{1}=k_{-1} \\
& \gamma_{1}=\gamma_{2}, \delta_{1}=\delta_{2}, k_{2}=k_{-2} \\
& k_{0}>k_{-0}
\end{aligned}
$$

(2) Active transfer of carrier-substrate complex:

$$
\begin{aligned}
& \alpha_{1}=\alpha_{2}, \beta_{1}=\beta_{2}, k_{1}>k_{-1} \\
& \gamma_{1}=\gamma_{2}, \delta_{1}=\delta_{2}, k_{2}>k_{-2} \\
& k_{0}=k_{-0}
\end{aligned}
$$

(3) Association-reaction type:

$$
\begin{aligned}
& k_{0}=k_{-0}, k_{1}=k_{-1}, k_{2}=k_{-2} \\
& \beta_{1}=\beta_{2}, \alpha_{1}>\alpha_{2} \\
& \delta_{1}=\delta_{2}, \gamma_{1}>\gamma_{2}
\end{aligned}
$$


(4) Dissociation-reaction type:

$$
\begin{aligned}
& k_{0}=k_{-0}, k_{1}=k_{-1}, k_{2}=k_{-2} \\
& \alpha_{1}=\alpha_{2}, \gamma_{1}=\gamma_{2} \\
& \beta_{2}>\beta_{1}, \delta_{2}>\delta_{1}
\end{aligned}
$$

In what follows, $C_{0}$ designates the total concentration of carrier in mass units per unit area of cell membrane. The latters $a, b, c, d, \ldots A, B, C, \ldots$ will be used to designate positive constants in exhibiting the functional form of the fluxes. However, they do not necessarily designate the same constants from equation to equation.

Transport fux. For the general case the transport flux is given by the following equation.

where

$$
F_{1}\left(u_{1}, v_{1}\right)=\frac{C_{0}}{\Delta}\left[\beta_{2} k_{0} k_{1} \alpha_{1} u_{1}-\beta_{1} k_{-0} k_{-1} \alpha_{2} v_{1}\right]
$$

$$
\begin{aligned}
\Delta=\left(k_{0}+k_{-0}\right)\left(\beta_{1} k_{-1}+\right. & \left.\beta_{2} k_{1}+\beta_{1} \beta_{2}\right)+\alpha_{1} u_{1}\left[\beta_{2}\left(k_{1}+k_{0}\right)+k_{0}\left(k_{-1}+k_{1}\right)\right] \\
& +\alpha_{2} v_{1}\left[\beta_{1}\left(k_{-1}+k_{-0}\right)+k_{-0}\left(k_{-1}+k_{1}\right)\right]+\alpha_{1} \alpha_{2} u_{1} v_{1}\left(k_{-1}+k_{1}\right)
\end{aligned}
$$

If we add to this the flux due to simple permeability, letting $k_{\mathrm{p}}$ be the permeability constant for the free amino acid, the net flux is given by a formula of the form

$$
\text { Flux }=\frac{a u_{1}-b v_{1}}{A+B u_{1}+C v_{1}+D u_{1} v_{1}}+k_{\mathbf{p}}\left(u_{1}-v_{1}\right)
$$

in which all constants are positive. The particular form of this equation for the different types of models can be obtained by making the appropriate substitutions for the defining conditions on the rate and transfer constants in Eqns. I and 2. Active transport is often treated as though Michaelis-Menten kinetics applied. Considering the form of Eqn. 3 we should not be surprised to find many deviations from MichaelisMenton kinetics, particularly since the intracellular concentrations attained in studies of initial flux are often considerably higher than extracellular at low extracellular concentrations.

Eqn. 3 can also be written as the difference of two one-way fluxes. Let $\overrightarrow{F_{1}}$ and $\langle$. represent the one-way fluxes into and out of the cell, respectively. Then these are given by Eqns. 4 and 5 .

$$
\begin{aligned}
& \overrightarrow{F_{1}}=\left(k_{\mathbf{p}}+\frac{a}{A+B u_{1}+C v_{1}+D u_{1} v_{1}}\right) u_{1} \\
& \check{F}_{1}=\left(k_{\mathbf{p}}+\frac{b}{A+B u_{1}+C v_{1}+D u_{1} v_{1}}\right) v_{1}
\end{aligned}
$$

In the steady state, attained when $u_{1}$ and $v_{1}$ remain constant, these are equal. Thus for the steady state, Relations 6 and 7 hold.

$$
\begin{gathered}
\frac{v_{1}}{u_{1}}=\mathrm{I}+\frac{a-b}{k_{\mathbf{p}}\left[A+b / k_{\mathrm{p}}+B u_{1}+C v_{1}+D u_{1} v_{1}\right]} \\
\frac{u_{\mathbf{1}}}{v_{1}-u_{1}}=\frac{k_{\mathrm{p}}}{a-b}\left[\left(A+b / k_{\mathrm{p}}\right)+B u_{1}+C u_{1}+D u_{1} v_{1}\right]
\end{gathered}
$$


Flux ir competition. For the situation in which both amino acids "I" and "2" are present at constant concentrations in the extracellular fluid but not intracellularly, this model predicts a relationship of the type shown in Eqn. 8.

$$
F_{1}\left(u_{1}, \mathrm{o}, u_{2}, \mathrm{o}\right)=\frac{a u_{1}}{A+B u_{1}+C u_{2}}
$$

The constant terms in this formula are extremely involved functions of the different rate and transfer constants. This model does not predict the stimulation obtained in some "competition" experiments. It predicts only increasing inhibition as $u_{2}$ increases, the actual degree of inhibition depending on the relative values of the constants $C$ and $A$ and $B$.

Flux in exchange diffusion. In the exchange-diffusion situation in which $u_{2}=0$ and $v_{1}=0$ and $u_{1}$ and $v_{2}$ are constant but non-zero, the equation obtained is of the form:

$$
F_{1}\left(u_{1}, \mathrm{o}, \mathrm{o}, v_{2}\right)=\frac{u_{1}\left(a+b v_{2}\right)}{A+B u_{1}+C v_{2}+D u_{1} v_{2}}
$$

The actual values for the constants in Eqn. 9 have been published ${ }^{11}$ (using a slightly different notation for the transfer constants). Depending on the relative values of the constants, this equation can predict an increase or decrease in the transport flux of amino acid " $\mathrm{I}$ " due to preloading with amino acid " 2 ".

Passive transport. If the transport is a passive transport (facilitated diffusion) without linkage to cellular metabolism and we can assume complete symmetry in the reaction rates and transfer processes, the equation for the transport flux when one amino acid is present simplifies considerably. In this case

$$
k_{0}=k_{-0}, k_{1}=k_{-1}, \alpha_{1}=\alpha_{2}, \beta_{1}=\beta_{2}
$$

The transport flux now becomes,

$$
F\left(u_{1}, v_{1}\right)=\frac{C_{0}}{\Delta} k_{0} k_{1} \beta_{1} \alpha_{1}\left(u_{1}-v_{1}\right)
$$

where $\Delta$ is now given by Eqn. II.

$$
\Delta=2 k_{0} \beta_{1}\left(2 k_{1}+\beta_{1}\right)+\alpha_{1}\left[k_{0}\left(\beta_{1}+2 k_{1}\right)+k_{1} \beta_{1}\right]\left(u_{1}+v_{1}\right)+2 k_{1} \alpha_{1}{ }^{2} u_{1} v_{1}
$$

The equations corresponding to Eqns. 8 and $g$ do not simplify appreciably.

\section{Carrier transport model; $I: I$ stoichiometry with exchange reaction}

If an exchange reaction can occur at each surface of the cell membrane, the model shown in Fig. I must be expanded to include the reaction shown in Eqn. I2.

$$
C S_{2}+S_{1} \underset{\varkappa_{2}}{\stackrel{\varkappa_{1}}{\rightleftharpoons}} C S_{1}+S_{2}
$$

This introduces an exchange reaction in which free carrier does not occur as an intermediate. The detailed formulation of such an exchange reaction would probably include the transient formation of a ternary complex ${ }^{13}$. It should be clearly distinguished from exchange diffusion although it could be an important contributing process to the increase in flux due to exchange diffusion. GUROFF has recently suggested in a private communication that an exchange reaction might explain the stimulation found in some competition experiments and CHRISTENSEN et al.14 had previously 
suggested that exchange reactions (displacement reactions in their terminology) might play a role in exchange diffusion.

We proceed then to present the implications of this. The model is the same as the one shown in Fig. I with the addition of reaction 12 at each surface.

Transport. If only amino acid "I" is present, the equation for the transport flux remains the same as Eqn. I. Thus no higher-order terms are introduced into the equation for the transport flux. This may be a significant feature as will be seen when we consider a model which involves 2 : I stoichiometry.

"Competition". If $u_{1}$ and $u_{2}$ are constant, and $v_{1}$ and $v_{2}$ are maintained at zero, the equation for the flux assumes the form:

$$
F_{1}\left(u_{1}, \mathrm{o}, u_{2}, \mathrm{o}\right)=\frac{u_{1}\left(a+b u_{1}+c u_{2}\right)}{A+B u_{1}+C u_{2}+D u_{1} u_{2}+E u_{1}^{2}+F u_{2}^{2}}
$$

The constants are so related that this reduces to the form:

$$
F_{1}\left(u_{1}, \mathrm{o}, \mathrm{o}, \mathrm{o}\right)=\frac{a u_{1}}{A+B u_{1}} \quad\left(\text { when } \mathrm{u}_{2}=\mathrm{o}\right)
$$

The functional dependence of Eqn. 13 on $u_{2}$ is such that $F_{1}\left(u_{1}, 0, u_{2}\right.$, o) could show either inhibition or stimulation and then inhibition with increase in $u_{2}$, depending on the values of the constants in Eqn. I3. Thus this model has the possibility of predicting the stimulation of uptake found in some experiments on competition ${ }^{3,5,6}$.

Exchange-diffusion flux. The exchange-diffusion flux in the presence of exchange reactions assumes the form:

$F_{1}\left(u_{1}, \mathrm{o}, \mathrm{o}, v_{2}\right)=\frac{u_{1}\left(a+b v_{2}\right)\left(c+d u_{1}+e v_{2}+f u_{1} v_{2}\right)}{A+B u_{1}+C v_{2}+D u_{1} v_{2}+E u_{1}^{2}+F v_{2}^{2}+G u_{1}^{2} v_{2}+H u_{1} v_{2}^{2}}$

The constants are such involved expressions in the rate and transfer constants, little is gained by exhibiting their detailed form. Again this clearly can predict an increase in flux due to exchange diffusion.

\section{Reversible adsorption transport models}

This is a limiting case of the previous carrier models. If we let the transfer rate constants increase, we obtain, in the limit, the adsorption-type model. In this case the total amount of carrier is in effect accessible to substrate from either side of the membrane. Since the transfer reactions occur at infinite speed, there are only two possible types of this model, the association-reaction type and the dissociationreaction type. A diagram of this model is shown in Fig. 2. We consider this model with and without the occurrence of exchange reactions.

Transport flux. Whether or not an exchange reaction occurs, the transport flux is given by Eqn. I6.

$$
F_{1}\left(u_{1}, v_{1}\right)=\frac{C_{0}\left(\beta_{2} \alpha_{1} u_{1}-\beta_{1} \alpha_{2} v_{1}\right)}{\beta_{1}+\beta_{2}+\alpha_{1} u_{1}+\alpha_{2} v_{1}}
$$

Note that this differs from the corresponding equation for carrier transport models (Eqn. 2) in not having a term involving the cross product $u_{1} v_{1}$ in the denominator.

Competition fux. If the exchange reaction does not occur, the flux of amino acid " $I$ " is given by Eqn. I7.

$$
F_{1}\left(u_{1}, \mathrm{o}, u_{2}, \mathrm{o}\right)=\frac{C_{0} \beta_{2} \alpha_{1} u_{1}\left(\delta_{1}+\delta_{2}\right)}{\left(\delta_{1}+\delta_{2}\right)} \frac{\left[\beta_{1}+\beta_{2}+\alpha_{1} u_{1}\right]+\left(\beta_{1}+\right) \beta_{2} \gamma_{1} u_{2}}{2}
$$


This shows only increasing inhibition with increase in $u_{2}$. However, if an exchange reaction can occur, $F_{1}\left(u_{1}, o, u_{2}, o\right)$ is given by Eqn. $\mathbf{I} 8$.

$$
\begin{gathered}
F_{1}\left(u_{1}, \mathrm{o}, u_{2}, \mathrm{o}\right)=\frac{C_{0} \beta_{2} u_{1}}{\Delta}\left[\alpha_{1}\left(\delta_{1}+\delta_{2}\right)+\varkappa_{1}\left(\alpha_{1} u_{1}+\gamma_{1} u_{2}\right)\right] \\
\Delta=\left(\dot{\beta}_{1}+\beta_{2}\right)\left(\gamma_{1} u_{2}+\varkappa_{1} u_{1}\right)+\left(\delta_{1}+\delta_{2}\right)\left(\beta_{1}+\beta_{2}+\alpha_{1} u_{1}+\varkappa_{2} u_{2}\right)+\left(\varkappa_{1} u_{1}+\varkappa_{2} u_{2}\right)\left(\alpha_{1} u_{1}+\gamma_{1} u_{2}\right)
\end{gathered}
$$

This is of the same functional form as Eqn. I3 and could explain stimulation of flux of amino acid " $I$ " in the presence of low concentrations of " 2 ".

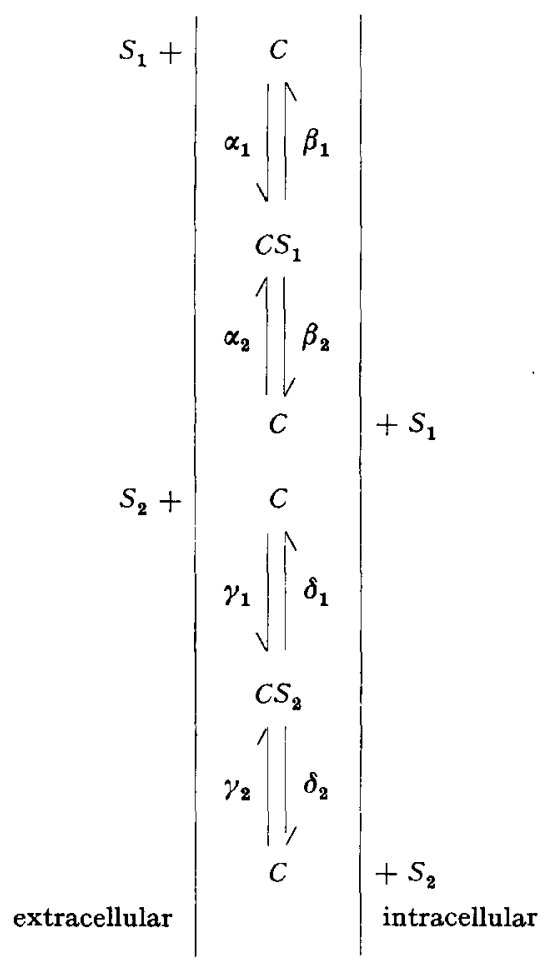

Fig. 2. Schematic diagram of adsorption transport model with two substrates $\left(S_{1}\right.$ and $\left.S_{2}\right)$.

Exchange diffusion. If there are no exchange reactions of the type of Eqn. I2, the flux of amino acid " $\mathrm{I}$ " in an exchange-diffusion situation is given by Eqn. 20.

$$
F_{1}\left(u_{1}, 0,0, v_{2}\right)=\frac{C_{0} \beta_{2}\left(\delta_{1}+\delta_{2}\right) \alpha_{1} u_{1}}{\left(\delta_{1}+\delta_{2}\right)\left[\beta_{1}+\beta_{2}+\alpha_{1} u_{1}\right]+\left(\beta_{1}+\beta_{2}\right) \gamma_{2} v_{2}}
$$

This predicts only a decrease in the flux of amino acid " $I$ " due to preloading with amino acid " 2 ". If exchange reactions occur, the exchange-diffusion flux takes the form of Eqn. $2 \mathrm{I}$.

$$
F_{1}\left(u_{1}, 0,0, v_{2}\right)=\frac{C_{0}}{\Delta}\left(\beta_{2}+\varkappa_{2} v_{2}\right)\left[\left(\delta_{1}+\delta_{2}\right) \alpha_{1} u_{1}+\varkappa_{1} u_{1}\left(\alpha_{1} u_{1}+\gamma_{2} v_{2}\right)\right]
$$

$$
\begin{gathered}
\Lambda=\left(\beta_{1}+\beta_{2}\right)\left(\delta_{1}+\delta_{2}\right)+\left(\beta_{1}+\beta_{2}\right) \gamma_{2} v_{2}+\left(\delta_{1}+\delta_{2}\right) \alpha_{1} u_{1}+\varkappa_{1} u_{1}\left(\beta_{1}+\beta_{2}+\alpha_{1} u_{1}+\gamma_{2} v_{2}\right) \\
+\varkappa_{2} v_{2}\left(\delta_{1}+\delta_{2}+\alpha_{1} u_{1}+\gamma_{2} v_{2}\right)
\end{gathered}
$$


This does predict the possibility of an increase in flux due to exchange diffusion. The specific forms these equations take for the two types of models may be obtained by letting $\beta_{1}=\beta_{2}$ and $\delta_{1}=\delta_{2}$ for the association-reaction type of model and by letting $\alpha_{2}=\alpha_{1}$ and $\gamma_{1}=\gamma_{2}$ for the dissociation-reaction type.

Passive transport. The equation for the net transport flux of one amino acid becomes particularly simple in a passive transport situation as shown by Eqn. 23 .

\section{Equilibrium-reaction models}

$$
F_{1}\left(u_{1}, v_{1}\right)=\frac{C_{0}\left(u_{1}-v_{1}\right)}{\left(2 / \alpha_{1}\right)+\left(\mathrm{I} / \beta_{1}\right)\left(u_{1}+v_{1}\right)}
$$

Another limiting case of the I: I carrier transport model is obtained if we allow the reaction rates to increase without bound. In the limit, the reactions become instantaneous and we can use equilibrium constants instead of reaction rates to describe the combination with carrier. The models worked out by RosENBERG AND WILBRANDT $^{\mathbf{1 2}}$ are all essentially of this type. A diagram of this model is given in Fig. 3 .

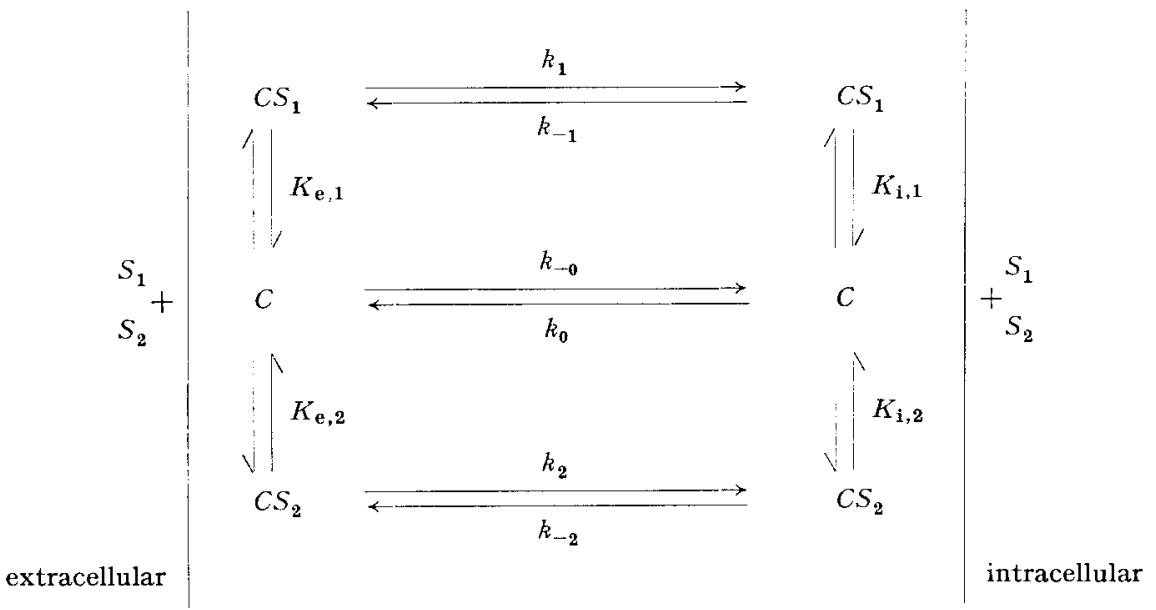

Fig. 3. Schematic diagram of general equilibrium-reaction model with $I:$ I carrier-substrate stoichiometry. $S_{1}$ and $S_{2}$ are two different substrates.

The equilibrium constants will be defined as association constants for the reactions given in Eqn. 24.

$$
\begin{aligned}
& S_{1}+C \rightleftharpoons C S_{1} \\
& S_{2}+C \rightleftharpoons C S_{2}
\end{aligned}
$$

Using the subscript $i$ to denote the inner surface of the membrane and e for the outer surface, the association constants are given by Eqn. 25.

$$
\begin{aligned}
K_{\mathrm{e}, 1}=\frac{\left[C S_{1}\right]_{\mathrm{e}}}{[C]_{\mathrm{e}} u_{1}} & K_{\mathrm{i}, 1}=\frac{\left[C S_{1}\right]_{\mathrm{i}}}{[C]_{\mathrm{i}} \nu_{1}} \\
K_{\mathrm{e}, 2}=\frac{\left[C S_{2}\right]_{\mathrm{e}}}{[C]_{\mathrm{e}}{u_{2}}^{2}} & K_{1,2}=\frac{\left[C S_{\mathbf{2}}\right]_{\mathrm{i}}}{[C]_{\mathbf{i}} v_{2}}
\end{aligned}
$$

For this model, we cannot define an independent exchange reaction since by the equilibrium assumption the equilibrium constants for the reactions

$$
S_{1}+C S_{2} \rightleftharpoons C S_{1}+S_{2}
$$


are given by Eqn. 26 .

$$
K_{\mathrm{e}}=\frac{K_{\mathrm{e}, 1}}{K_{\mathrm{e}, 2}} \quad K_{1}=\frac{K_{1,1}}{K_{\mathrm{i}, 2}}
$$

The four different types of this model are given by the following conditions. The association-reaction and dissociation-reaction types are no longer distinguishable in terms of inequality relations for the reaction parameters.

(I) Active transfer of free carrier:

$$
\begin{aligned}
& k_{1}=k_{-1}, k_{2}=k_{-2} \\
& K_{\mathrm{e}, 1}=K_{\mathbf{i}, 1}, K_{\mathrm{e}, 2}=K_{\mathbf{i}, 2} \\
& k_{0}>k_{-0}
\end{aligned}
$$

(2) Active transfer of carrier-substrate complex:

$$
\begin{aligned}
& k_{0}=k_{-0}, K_{\mathrm{e}, 1}=K_{\mathrm{i}, 1}, K_{\mathrm{e}, 2}=K_{\mathrm{i}, 2} \\
& k_{1}>k_{-1}, k_{2}>k_{-2}
\end{aligned}
$$

(3) Association-reaction type, dissociation-reaction type

$$
\begin{aligned}
& k_{0}=k_{-0}, k_{1}=k_{-1}, k_{2}=k_{-2} \\
& K_{\mathrm{e}, 1}>K_{\mathrm{i}, 1}, K_{\mathrm{e}, 2}>K_{1,2}
\end{aligned}
$$

Transport flux. The net transport flux if only amino acid " $\mathrm{I}$ " is present is given by $F_{1}\left(u_{1}, v_{1}\right)$

$$
=\frac{F_{1}\left(u_{1}, v_{1}\right) \quad C_{0}\left[k_{0} k_{1} K_{\mathrm{e}, 1} u_{1}-k_{-0} k_{-1} K_{\mathrm{i}, 1} v_{1}\right]}{\left(k_{0}+k_{-0}\right)+\boldsymbol{Y} K_{\mathrm{e}, 1}\left(k_{0}+k_{1}\right) u_{1}+K_{\mathbf{i}, 1}\left(k_{-0}+k_{-1}\right) v_{1}+K_{\mathrm{e}, 1} K_{1,1}\left(k_{-1}+k_{1}\right) u_{1} v_{1}}
$$

"Competition" flux. The flux obtained when $u_{1}$ and $u_{2}$ are constant and $v_{1}$ and $v_{2}$ are zero is given by Eqn. 28.

$$
F_{1}\left(u_{1}, o, u_{2}, o\right)=\frac{C_{0} k_{0} k_{1} K_{\mathrm{e}, 1} u_{1}}{\left(k_{0}+k_{-0}\right)+\left(k_{0}+k_{1}\right) K_{\mathrm{e}, 1} u_{1}+\left(k_{0}+k_{2}\right) K_{\mathrm{e}, 2} u_{2}}
$$

Thus the equilibrium carrier model predicts only an increasing inhibition of the flux of amino acid " $I$ " as the concentration of amino acid " 2 " increases and is therefore not appropriate for application to amino acid transport.

Flux in exchange diffusion. For the conditions $u_{2}=v_{1}=0$ and $u_{1}$ and $v_{2}$ constant, the flux of amino acid " $I$ " is given by

$$
F_{1}\left(u_{1}, \mathrm{o}, \mathrm{o}, v_{2}\right)=\frac{C_{0} k_{1} K_{\mathrm{e}, 1} u_{1}\left(k_{0}+k_{-2} K_{\mathrm{i}, 2} v_{2}\right)}{\left(\mathrm{I}+K_{\mathrm{e}, 1} u_{1}\right)\left(k_{0}+k_{-2} K_{\mathrm{i}, 2} v_{2}\right)+\left(k_{-0}+k_{1} K_{\mathrm{e}, 1} u_{1}\right)\left(\mathrm{I}+K_{\mathrm{i}, 2} v_{2}\right)}
$$

This equation predicts the possibility of stimulation of the flux of amino acid " $I$ " due to exchange diffusion since it has the functional form:

$$
F_{1}\left(u_{1}, \mathrm{o}, \mathrm{o}, v_{2}\right)=\frac{u_{1}\left(a+b v_{2}\right)}{A+B u_{1}+C v_{2}+D u_{1} v_{2}}
$$

Passive transport. For passive transport with $k_{0}=k_{-0}, k_{1}=k_{-1}^{\prime}, K_{\mathrm{e}, 1}=K_{1,1}$ $=K_{1}$, the transport flux is given by Eqn. 3 I.

$$
F_{1}\left(u_{1}, v_{1}\right)=\frac{C_{0} k_{0} k_{1} K_{1}\left(u_{1}-v_{1}\right)}{2 k_{0}+K_{1}\left(k_{0}+k_{1}\right)\left(u_{1}+v_{1}\right)+2 K_{1}^{2} k_{1} u_{1} v_{1}}
$$


Equilibrium-reaction model; $2:$ I substrate-carrier stoichiometry

Finally we consider what effects would be introduced if the carrier binds 2 molecules of substrate. This has only been worked out for the equilibrium carrier model of the type in which there is active transfer of free carrier. This is depicted in

extracellular
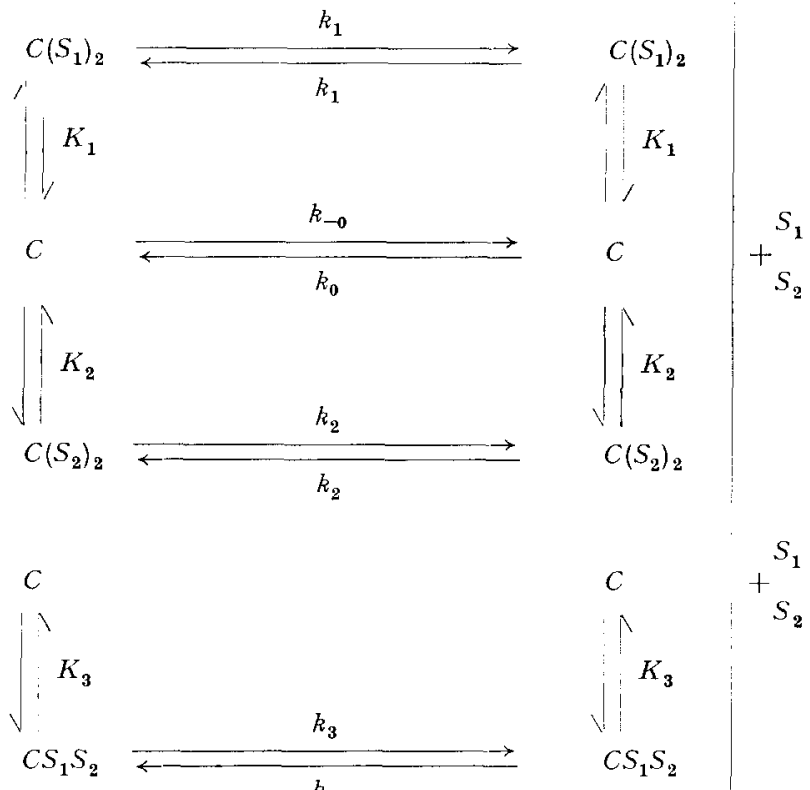

intracellular

Fig. 4. Schematic diagram for equilibrium-reaction model of the type with active transfer of free carrier. The stoichiometry is $2:$ I substrate-carrier binding ratio.

Fig. 4. The algebra for the general case becomes frightening. Some of the results for this model have already been published using a slightly different notation ${ }^{\mathbf{5}}$.

Transport flux. The transport flux is given by Eqn. (32).

$$
F_{1}\left(u_{1}, v_{1}\right)=\frac{2 C_{0} k_{1} K_{1}\left[k_{0} u_{1}^{2}-k_{-0} v_{1}^{2}\right]}{\left(\mathbf{I}+K_{1} u_{1}^{2}\right)\left(k_{0}+k_{1} K_{1} v_{1}^{2}\right)+\left(\mathbf{I}+K_{1} v_{1}^{2}\right)\left(k_{-0}+k_{1} K_{1} u_{1}^{2}\right)}
$$

This is second order in $u_{1}$ and $v_{1}$. This may be useful in distinguishing between $\mathbf{I}: \mathbf{I}$ and 2:I stoichiometry.

Competition fux. The flux in a competition situation is given by Eqn. 33 .

$$
\begin{gathered}
F_{1}\left(u_{1}, \mathrm{o}, u_{2}, \mathrm{o}\right)=\frac{C_{0} k_{0}}{\Delta}\left[2 k_{1} K_{1} u_{1}^{2}+k_{3} K_{3} u_{1} u_{2}\right] \\
\Delta=k_{0}+k_{-0}+K_{1}\left(k_{0}+k_{1}\right) u_{1}^{2}+K_{2}\left(k_{0}+k_{2}\right) u_{2}{ }^{2}+K_{3}\left(k_{0}+k_{3}\right) u_{1} u_{2}
\end{gathered}
$$

This clearly could give first an increase and then a decrease in the flux of amino acid " $I$ " as $u_{2}$ increases. 
Exchange diffusion. For $u_{2}=v_{1}=0$ and $u_{1}$ and $v_{2}$ constant, the flux of amino acid " $I$ " is given by Eqn. 34 .

$$
\begin{gathered}
F_{1}\left(u_{1}, \mathrm{o}, \mathrm{o}, v_{2}\right)=\frac{2 C_{0} k_{1} K_{1} u_{1}^{2}}{\Delta}\left(k_{0}+k_{2} K_{2} v_{2}{ }^{2}\right) \\
\Delta=k_{0}+k_{-0}+K_{1}\left(k_{0}+k_{1}\right) u_{1}{ }^{2}+K_{2}\left(k_{-0}+k_{2}\right) v_{2}{ }^{2}+K_{1} K_{2}\left(k_{1}+k_{2}\right) u_{1}{ }^{2} v_{2}{ }^{2}
\end{gathered}
$$

This clearly can predict a stimulation of uptake of one amino acid after preloading with another amino acid.

\section{DISCUSSION}

It is evident that all of the experimental findings on amino acid active transport in Ehrlich ascites cells can be predicted at least qualitatively either with a model having a $I$ : I stoichiometry and which admits exchange reactions at the membrane surfaces or a model which has a $2: 1$ amino acid-carrier stoichiometry. It is possible that these two models might be distinguished by determining whether the transport flux shows a first or second-order dependence on extracellular concentration but it seems more likely that detailed computational studies will be required.

\section{ACKNOWLEDGEMENTS}

This work was supported in part by grants GM-892 and CA-06734 from the National Institutes of Health, U.S. Public Health Service.

\section{REFERENCES}

1 H. N. Christensen, T. R. Riggs, H. Fischer and I. M. Palatine, J. Biol. Chem., 198 (I952) I.

${ }^{2}$ E. HeInz, J. Biol. Chem., 2 I I (I954) $78 \mathrm{I}$.

3 J. A. Jacquez, Am. J. Physiol., 200 (196I) 1063.

4 H. N. Christensen and T. R. Riggs, J. Biol. Chem., I94 (I952) 57.

5 J. A. JacQUez, Biochim. Biophys. Acta, 7 I (I963) I5.

6 M. A. Chirigas, G. R. Fanning and G. Guroff, Cancer Res., 22 (I962) I 349.

7 E. Heinz and P. M. Walsh, J. Biol. Chem., 233 (I958) 1488.

8 R. M. Johnstone and P. G. Scholefield, J. Biol. Chem., 236 (I96I) I4I9.

9 R. M. Johnstone ANd J. H. Quastel, Biochim. Biophys. Acta, 46 (I96I) 527.

10 T. Rosenberg and W. Wilbrandt, J. Gen. Physiol., 4I (I957) 289.

11 J. A. Jacguez, Proc. Natl. Acad. Sci. U.S., 47 (Ig6I) I 53.

12 T. Rosenberg and W. Wilbrandt, Exptl. Cell Res., 9 (I955) 49.

13 J. Tze-Fei Wong and C. S. Hanes, Can. J. Biochem. Physiol., 40 (1962) 763.

14 H. N. Christensen, H. Akedo, D. L. Oxender and C. G. Winter, Amino Acid Pools, Elsevier, Amsterdam, I962, p. 527. 